\title{
The journey from excellence to innovation
}

\author{
Melinda Kenneway \\ Executive Director, Kudos Innovation Ltd, Wheatley, Oxfordshire, UK \\ E-mail: Melinda@GrowKudos.com; URL:GrowKudos.com
}

\begin{abstract}
Once you have understood that the focus on excellence may hamper innovation, you can focus much better on bringing an innovation to market that customers want. As a start-up founder, I have learnt that you simply don't have the resources or time to focus on anything but a real market need for delivering innovation. Looking ahead, more opportunities for founders in academic publishing may be identified, e.g. in improving access, sharing data, and enhancing the speed of publication.
\end{abstract}

Keywords: Innovation, excellence, publishing, startup, business model

\section{Innovation is hard}

I have been in the academic publishing industry for more than twenty years in various roles. What I've learned in that time, and most particularly in my role as Executive Director of a start-up, is that innovation is very difficult to do. Coming from a traditional background there are many things that I held sacred relating to products and processes. Setting up and building a fast-growth start-up requires you to put some of those sacred things to one side. You simply don't have the resources or time to do things in the same way as more established organizations. Not only that, your very purpose must be sufficiently innovative and meet a real market need, or you won't have much of a future.

Today I want to report on my journey but also reflect on the wider landscape. Letting go of the concept of excellence was a difficult but essential lesson for me, because the commitment to excellence easily hampers innovation. So, I first want to expand on this, and then look at innovation in publishing in five key areas: access, data, speed, utility, and impact.

\section{Excellence hampers innovation}

Excellence is prized in academic publishing. It is uncontroversial - it's easy to have the moral high ground when you focus on excellence. By contrast, innovation requires you to challenge and change things. As Thomas and Sara DeLong noted, the paradox of excellence is that for high achievers, looking stupid or incompetent is anathema. So, they stick to the tasks they're good at. In short, they'd rather do the wrong thing well than do the right thing poorly [1].

Excellence is everywhere. It is used to describe the desirable outcome in research, teaching, and publishing. But what is the cost of excellence? Clearly, excellence is very expensive in terms of money and time spent. What is the trade off in terms of the value we are getting? 


\section{The cost of excellence}

Samuel Moore and colleagues noted the following: "A focus on excellence impedes rather than promotes scientific and scholarly activity: it at the same time discourages both the intellectual risk-taking... and the careful 'normal science' that allows us to consolidate our knowledge" [2].

Striving for excellence also means seeking to avoid failure, and being afraid of failure. This has a cost too. Compare aviation and healthcare. In the United States, there is only 1 accident for more than two million flights and not very many people die. In healthcare, however, more than 400,000 people die every year prematurely due to medical errors. What creates this difference? In aviation, you have a global open loop approach to safety and innovation, leading to changes and improvements after each accident. In medicine, we have a closed and local system to quality and safety, leading to much less innovation.

\section{Innovation drives economic progress and well-being}

Imagine that you are launching a startup company, without legacy business models and established revenue streams to worry about. If you are not vested in the status quo, innovation is easier to do. Let us also imagine that researchers are rewarded not for excellence but for driving innovation. How would that change our focus? What kind of opportunities might this open up?

1. A culture of access that enables the easy sharing and mining of articles and data. For that, innovation is required not only at level of infrastructure and regulation, but also efforts at enriching content with better metadata that help bridge silos and make more content easily accessible.

2. A black box culture for data that encourages much more recording and sharing of negative results as well as study replication. Equally important are incentives for the immediate release of data, for example, by enhancing standards and citation practices.

3. Speed is becoming much more important, possibly to the point where speed of publication is more important than journal brand value. Accelerated publication and digestible formats is not the endall however, because further acceleration of the research cycle requires that contextual relevance and semantic discovery is enhanced.

4. Utility is another opportunity, and centres on impact and application. This means developing and defining research questions with the eventual downstream audience in mind. This could be a research-user perspective but equally based on the grand challenges humanity faces in the $21 \mathrm{st}$ century.

5. We need to be thinking about impact in a different way if we are to encourage innovation. Our current, publication-focused system is heavily weighted towards those researchers more established in their careers and is a disincentive to conducting original, creative, and transformative research. We need a new system that incentivizes behaviours that accelerate impact and support universities in producing well-developed and creative people with the capabilities to innovate in a world of open research.

\section{References}

[1] T. DeLong and S. DeLong, 'Managing Yourself: The Paradox of Excellence', Harvard Business Review, June 2011, https:// hbr.org/2011/06/managing-yourself-the-paradox-of-excellence.

[2] S. Moore et al., Excellence R us: University research and the fetishisation of excellence, Palgrave Communications 3(4) (2016), 16105. doi:10.1057/palcomms.2016.105. 